\title{
Adaptation to the dominance tube
}

WAYNE J. WILSON

STEPHEN F. AUSTIN STATE COLLEGE

Two groups of rats inexperienced in dominance tube competition opposed respectively an established group of dominant and submissive Ss. Results were negligible between dominant and naive Ss but submissive animals performed below chance against naive opponents, after displaying transitory ascendance during early dominance contests. A fifth group of rats was used to demonstrate the reliability of tube dominance over an extended period of time.

Candland \& Bloomquist (1965) have reported low reliability in achieving a dominance order involving rats (Carworth CBE) jockeying for possession of reward in a situation which resembled the food-hole task devised by Uyeno (1960). However, other investigators have been able to derive a stable hierarchy involving inbred strains of mice and rats, respectively, by using the dominance tube, a task which forces an animal to push an opponent through a tube too narrow for either $S$ to pass unhindered (Lindzey, Winston, \& Manosevitz, 1961; Lindzey, Manosevitz, \& Winston, 1966; Schumsky \& Jones, 1966; Uyeno \& White, 1967). These findings indicate the dominance tube to be useful for generating artificial rodent hierarchies on demand, but there is a scarcity of normative data by which to estimate the absolute utility value of this task. The present experiment is designed, first, to determine the reliability of tube dominance over an extended number of days, and, second, to assess the adaptability of relatively naive rats paired competitively with established dominant and submissive opposition. Nethed

The Ss were 60 male Sprague-Dawley rats, approximately 90 days old at the time of experimentation. Fortytwo Ss were obtained from Sprague-Dawley, Inc., and socially isolated upon arrival, and $18 \mathrm{Ss}$ were reared in the animal colony of Texas Christian University and housed individually after weaning. The latter animals were distributed randomly among the five groups of 12 rats each used in the experiment.

The dominance apparatus consisted of a Lucite tube, $2-1 / 2 \times 24$ in. The tube was connected at each end to a start box which had a width of 8 in. tapering to 3 in. at the rear, with length and height dimensions of $7 \times 5$ in. A goal box measuring $10 \times 3 \times 5$ in. was attached to each start compartment and both were covered with Plexiglas. Guillotine doors were located at the entrance of each start box and goal box and a guillotine divider was inserted midway in the tube. Starting times were initiated when the raising of either start door released a microswitch, and photocells were located 2-1/4 in. from the outside entrance of each start box and 1 in. on either side of the tube divider in order to distin- guish start times and running times (to the midpoint of the tube). Standard Electric timers recorded times in $.001 \mathrm{~min}$.

Three of five groups were placed under $23 \mathrm{~h}$ water deprivation and gentled for seven days. Adaptation to the dominance tube consisted of $5 \mathrm{~min}$ solo exploration on Day 1 and a rewarded trial in both directions on Day 2, with a criterion of $60 \mathrm{sec}$ contact drinking or a $5 \mathrm{~min}$ confinement period required after each trial. On Days 3 to 8 each $S$ received five massed, reinforced trials (15 sec permitted in goal box) per day with the direction of passage randomized but with an equal number of rewards given in each end box at the conclusion of 30 trials.

One group was then designated to demonstrate the reliability of tube dominance for 39 days (13 replications). Each $S$ in this group received three to four dominance contests per day until every member had been encountered once over a three-day cycle. The two remaining groups were combined and given a schedule of dominance matches similar to the first group's, except that six days were required for a replication. When three replications were obtained, the combined group was dichotomized into 12 dominant (Group D) and 12 submissive (Group S) animals based on power scores derived from a dominance matrix (Kemeny, Snell, \& Thompson, 1957).

The two excluded groups completed pretraining and training at a time to coincide with the termination of the final replication used to extablish Groups D and S. The two naive groups were paired respectively with Groups $D$ and $S$ for 18 test days, each $S$ receiving two dominance contests per day with six days required for a replication. During all matching trials, each $\mathrm{S}$ was allowed a solo reward run preliminary to the dominance contest. Ss were then released simultaneously into the tube and a win declared when one $S$ forced another back until its hind-feet touched the opposite start box; neither $S$ was given a reward and both were removed immediately from the apparatus. It should be noted that in the total experimental procecure, naive animals received less handling and a smaller number of rewards than did Ss of Groups D and S.

\section{Results and Discussion}

The first group assigned to engage in an extended number of matching trials yielded reliable dominance orders across replications as indexed by a series of rho coefficients for adjacent replications (Fig. 1). One set of correlations was based on ranks derived from contests won and a set was represented by ranks converted from power scores, the latter measures being shown as comparable to the first (in this instance) 


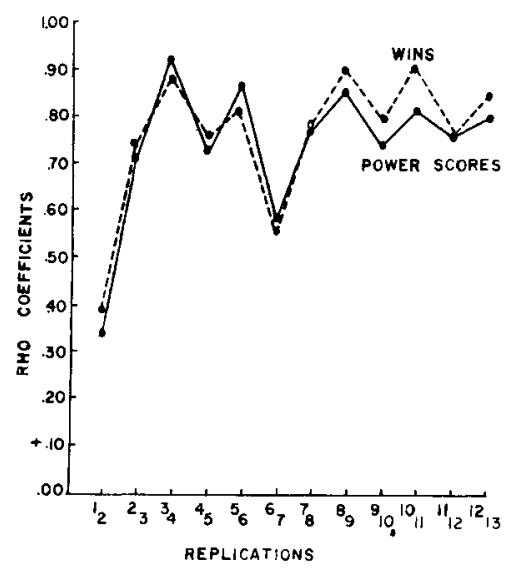

Fig. 1. Rank order coefficients used to show reliability of tube dominance orders based on frequency of wins and on power scores.

but more informative since the rank of those animals defeated by a dominant $\mathrm{S}$ is taken into consideration. Times measures were not graphed in this report, but start times and tube running times were slower for all Ss on matching trials than on preliminary reinforced trials, and faster running times on both types of trials were noted for dominant Ss (top six ranks) as compared to submissive Ss (bottom six ranks) after the fourth of 13 replications.

Data pertaining to the adaptability of naive opposition were analyzed by the Wilcoxon Test for Matched Pairs, comparing obtained wins against chance performance during the final replication for each group. Chance performance was adjusted to accommodate the dominance level of each established group, i.e., $75 \%$ wins expected for Group D and 25\% expected for Group S. Results were not significant for Group D but were decisive for Group S, which won only eight rather than the expected 36 of their final 144 contests $(T=0 ; n=12 ; p<.01)$. Figure 2 illustrates the decline in dominance of Group $S$ which is marked after Day 4 (after an accrual of eight dominance contests by naive Ss). A 2 by 2 factorial assessed the final three matching trials and indicated faster speeds for Group $D$ than Group $S$ on median starting latencies $(F=7.77 ; d f=1 / 44 ; p<.01)$ and median running times $(F=12.00 ; d f=1 / 44 ; p<.01)$. Significant interaction effects for median starting times $(F=5.92$; $\mathrm{df}=1 / 44 ; \mathrm{p}<.05)$ and median running times $(\mathrm{F}=16.87$; $\mathrm{df}=1 / 44 ; \mathrm{p}<.01$ ) point to negligible speed differences between naive groups, which were positioned intermediate to the faster speeds of Group $D$ and slower times of Group S. Differences between the four conditions on preliminary reinforced trials were not significant.

Stability in the dominance order across 13 replications was supported by the high reliability coefficients illustrated in Fig. 1, demonstrating that tube dominace can be a useful measure when a $S$ engages in three to four contests per day over an extended period of time.

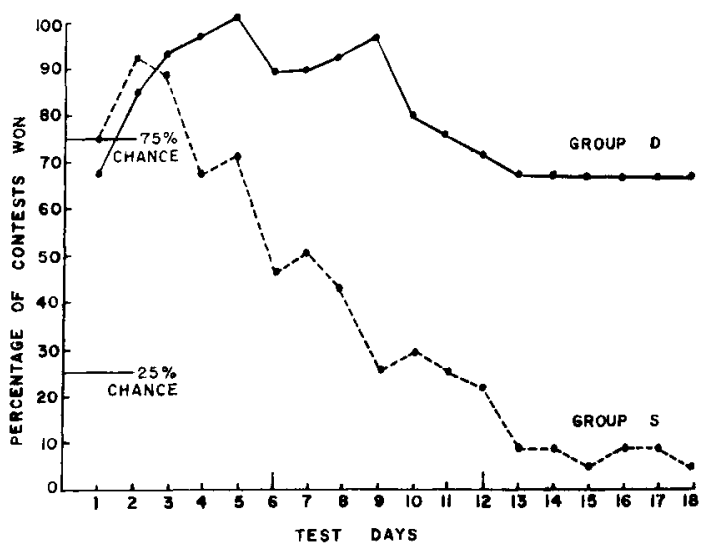

Fig. 2. Percentage of contests won by Groups $D$ and $S$ against naive opposition. Note that the adjusted chance performance is $75 \%$ wins expected for Group D and 25\% for Group S.

Data related to the pairing of naive Ss against Group D are nominal but findings for Group $S$ suggest that a high frequency of prior defeats could condition these Ss to be submissive in the tube, despite the fact that the opposition had less combative experience, was handled less frequently, and received a smaller number of reinforcements in the total experimental plan.

Schumsky \& Jones (1966) failed to uncover a relationship between running speed and dominance order in their investigation; however, the exact nature of their time measurement was not specified and there is obviously support for such a relationship in the present experiment. Furthermore, these authors suggested that the level of aggressiveness may be pre-experimentally determined. If so, then one might estimate that by chance $25 \%$ of Group S should have retained dominance (but did not) over a random sample of naive Ss. The point is that a fixed dominance level need not emerge automatically, for its expression could be modified by the functioning of an adversive emotional component.

\section{References}

CANDLAND, D., \& BLOOMQUIST, D. Interspecies comparisons of the reliability of domince orders. J. comp. physiol Psychol., 1965, 59, 135-137.

KEMENY, J., SNELL, J., \& THOMPSON, G. Introduction to finite mathematics. Englewood Cliffs: Prentice-Hall, 1957.

LINDZEY, G., WINSTON, H., \& MANOSEVITZ, M. Social dominance in inbred mouse strains. Nature, 1961, 191, 474-476.

LINDZEY, G., MANOSEVITZ, M., \& WINSTON, H. Social dominance in the mouse. Psychon. Sci, 1966, 5, 451-452.

SCHUMSKY, D., \& JONES, P. Reliable paired comparison dominance orders in rats. Psychol. Rec., 1966, 16, 473-478.

UYENO, E. Hereditary and environmental aspects of dominance behavior in the albino rat. J. comp. physiol. PsychoL, 1960, 53, 138-141. UYENO, E., \& WHITE, M. Social isolation and dominance behavior. $J$. comp. physiol. Psychol. 1967, 63, 157-159.

Note

1. Data was collected in the summer of 1963 when the author was serving on a National Science Foundation Summer Teaching Fellowship at Texas Christian University. Thanks are due to Winton Manning for his assistance concerning applications of the dominance matrix. 